\title{
A Sampling Theory for Compact Sets in Euclidean Space
}

\author{
Frédéric Chazal · David Cohen-Steiner • \\ André Lieutier
}

Received: 30 August 2006 / Revised: 20 June 2007 / Accepted: 20 June 2007 /

Published online: 19 February 2009

(C) Springer Science+Business Media, LLC 2009

\begin{abstract}
We introduce a parameterized notion of feature size that interpolates between the minimum of the local feature size and the recently introduced weak feature size. Based on this notion of feature size, we propose sampling conditions that apply to noisy samplings of general compact sets in euclidean space. These conditions are sufficient to ensure the topological correctness of a reconstruction given by an offset of the sampling. Our approach also yields new stability results for medial axes, critical points, and critical values of distance functions.
\end{abstract}

Keywords Distance function - Sampling - Surface and manifold reconstruction

\section{Introduction}

In this paper, we use the framework of distance functions to study some geometric and topological approximation problems on compact subsets of $\mathbb{R}^{n}$.

The authors were partially supported by DARPA under grant HR0011-05-1-0007 and by ANR under grant "Topologie, Géométrie Différentielle et Algorithmes."

F. Chazal

INRIA Futurs, 2-4 rue Jacques Monod, 91893 Orsay Cedex, France

e-mail: Frederic.Chazal@inria.fr

D. Cohen-Steiner $(\varangle)$

INRIA Sophia-Antipolis, 2004, route des lucioles, 06902 Sophia-Antipolis Cedex, France e-mail: David.Cohen-Steiner@sophia.inria.fr

\section{A. Lieutier}

Dassault Systèmes and LMC/IMAG, Grenoble, France

e-mail: andre_lieutier@ds.fr 


\subsection{Motivation}

In many practical situations, the object of study is only known through a finite set of possibly noisy sample points. It is then desirable to try to recover the geometry and the topology of the object from this information. The most obvious example is probably surface reconstruction, where the points are measured on the surface of a real world object. Also, a current research topic in cosmology is to study the large-scale structure formed by the galaxies, which seems to be an interconnected network of walls and filaments [27]. In other applications, the shape of interest may live in a higherdimensional space, as for instance in machine learning and in particular in manifold learning. This is also the case in time series analysis, when the shape of study is the attractor of a dynamical system sampled by a sequence of observations [25].

In this context, an important question is to find a sampling condition guaranteeing that the object can be reconstructed correctly. Besides providing theoretical guarantees, such a condition may be used to drive the reconstruction process itself. Indeed, a possible reconstruction strategy is to look for the shapes that are best sampled by the data points. In what follows, we investigate these questions in a fairly general setting, assuming a very simple reconstruction process.

\subsection{Previous Work}

The currently most successful framework for dealing with such problems is based on the notion of $\varepsilon$-sample introduced by Amenta et al. [1]. A sampling of a shape $K$ is an $\varepsilon$-sampling if every point $p$ in $K$ has a sample point at distance at most $\varepsilon \operatorname{lfs}_{K}(p)$, where $\operatorname{lfs}_{K}(p)$ denotes the local feature size of $p$, that is, the distance from $p$ to the medial axis of the complement of $K$. It has been shown that surfaces smoothly embedded in $\mathbb{R}^{3}$ can be reconstructed homeomorphically from any 0.06-sampling using the Cocone algorithm [2]. The major limitation of the $\varepsilon$-sample framework is that it cannot handle sharp edges. Indeed, the local feature size vanishes on such edges, implying that any $\varepsilon$-sample must have an infinite number of points in their vicinity. Boissonnat et al. [3] recently gave sampling conditions applying to nonsmooth surfaces in $\mathbb{R}^{3}$ and guaranteeing that the restricted Delaunay triangulation of the surface is isotopic to the surface. Yet, to the best of our knowledge, no reconstruction algorithm comes with theoretical guarantees in the case of nonsmooth shapes, except for curves [13].

One of the simplest methods for reconstructing arbitrary shapes is to output an offset of the sampling for a suitable value $\alpha$ of the offset parameter. Topologically, this is equivalent to taking the $\alpha$-shape [17] of the data points, which can be computed efficiently in $\mathbb{R}^{3}$ using the Delaunay triangulation. Recently, Niyogi, Smale, and Weinberger [24] proved that this method indeed provides reconstructions having the correct homotopy type for densely enough sampled smooth submanifolds of $\mathbb{R}^{n}$. The precise sampling condition is that the Hausdorff distance between the sampling and the submanifold should not exceed $\sqrt{9}-\sqrt{8}$ times the minimum of the local feature size function over the submanifold. Note that this condition is similar to the $\varepsilon$-sampling condition, except that noise is allowed and local adaptivity to the variations of the local feature size is lost. It was recently shown that under a similar but 
locally adaptive condition, the union of balls centered on data points and with radius proportional to the local feature size is homotopy equivalent to the submanifold [7]. Both conditions suffer from the same problem as $\varepsilon$-samplings, namely they do not apply to shapes other than smooth submanifolds. For more general shapes, the homology groups can be estimated correctly from a sufficiently dense sampling using topological persistence techniques $[6,11]$, but it is not known how to actually build a reconstruction having the correct homology groups.

\subsection{Contributions}

In this paper, we introduce a parameterized set of sampling conditions that permit to extend the result of Niyogi, Smale, and Weinberger to a large class of compact subsets of euclidean space. Our sampling conditions resemble those used by Niyogi, Smale, and Weinberger, except that they are not based on the local feature size but rather on a parameterized notion of feature size that we call the $\mu$-reach. The $\mu$-reach of a compact set $K$ is the minimum distance between a point in $K$ and a point in the $\mu$-medial axis of $\mathbb{R}^{n} \backslash K$, which is a filtered version of the medial axis. In particular, the $\mu$-reach interpolates between the minimum of the local feature size (for $\mu=1$ ) and the weak feature size (for $\mu \rightarrow 0$ ). A crucial ingredient in our approach is a generalization of a result on the separation of the critical values of distance functions recently obtained by Dey et al. [15] in the smooth case. We also introduce the concept of critical function of a compact set, which can be used to choose the offset parameter appropriately by searching for the values that optimize the sampling quality. Along the way, we obtain new stability results for the $\mu$-medial axis, as well as for critical points, critical values, and for the critical function itself.

\subsection{Outline}

Section 2 recalls the necessary background on distance functions and their gradient and critical points. In Sect. 3, we use a key lemma on distance functions to derive an approximation result on $\mu$-medial axes. Section 4 introduces the critical function and the $\mu$-reach, and proves our generalization of the result of Niyogi, Smale, and Weinberger on offsets. Section 5 concludes the paper.

\section{Background on Distance Functions}

The distance function $R_{K}$ of a compact subset $K$ of $\mathbb{R}^{n}$ associates to each point $x \in \mathbb{R}^{n}$ its distance to $K$ :

$$
x \mapsto R_{K}(x)=\min _{y \in K} d(x, y),
$$

where $d(x, y)$ denotes the euclidean distance between $x$ and $y$. Conversely, this function characterizes completely the compact set $K$ since $K=\left\{x \in \mathbb{R}^{n} \mid R_{K}(x)=0\right\}$. Note that $R_{K}$ is 1 -Lipschitz. For a positive number $\alpha$, we denote by $K^{\alpha}$ the $\alpha$-offset of $K$ defined by $K^{\alpha}=\left\{x \mid R_{K}(x) \leq \alpha\right\}$. The Hausdorff distance $d_{H}\left(K, K^{\prime}\right)$ between 
Fig. 1 A two-dimensional example with two closest points

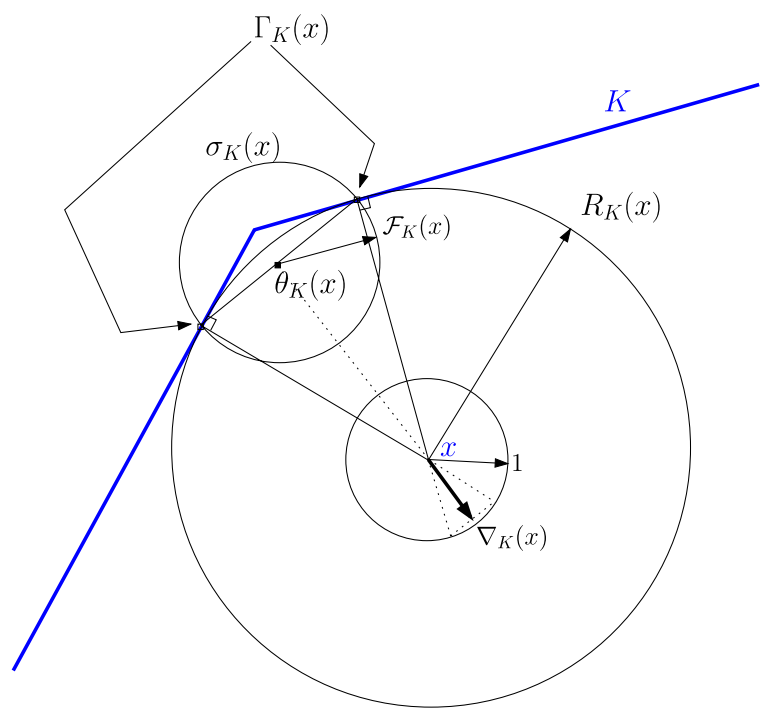

two compact sets $K$ and $K^{\prime}$ in $\mathbb{R}^{n}$ is the minimum number $r$ such that $K \subset K^{\prime r}$ and $K^{\prime} \subset K^{r}$. It is not difficult to check that the Hausdorff distance between two compact sets is the maximum difference between the distance functions associated with the compact sets:

$$
d_{H}\left(K, K^{\prime}\right)=\sup _{x \in \mathbb{R}^{n}}\left|R_{K}(x)-R_{K^{\prime}}(x)\right|
$$

\subsection{The Gradient and its Flow}

Given a compact subset $K$ of $\mathbb{R}^{n}$, its associated distance function $R_{K}$ is not differentiable on the medial axis of $\mathbb{R}^{n} \backslash K$. However, it is possible [22] to define a generalized gradient function $\nabla_{K}: \mathbb{R}^{n} \rightarrow \mathbb{R}^{n}$ that coincides with the usual gradient of $R_{K}$ at points where $R_{K}$ is differentiable. For any point $x \in \mathbb{R}^{n} \backslash K$, we denote by $\Gamma_{K}(x)$ the set of points in $K$ closest to $x$ (Fig. 1):

$$
\Gamma_{K}(x)=\{y \in K \mid d(x, y)=d(x, K)\} .
$$

Note that $\Gamma_{K}(x)$ is a nonempty compact set. There is a unique smallest closed ball $\sigma_{K}(x)$ enclosing $\Gamma_{K}(x)$ (cf. Fig. 1). We denote by $\Theta_{K}(x)$ the center of $\sigma_{K}(x)$ and by $\mathcal{F}_{K}(x)$ its radius. $\Theta_{K}(x)$ can equivalently be defined as the point on the convex hull of $\Gamma_{K}(x)$ nearest to $x$. For $x \in \mathbb{R}^{n} \backslash K$, the generalized gradient $\nabla_{K}(x)$ is defined as follows:

$$
\nabla_{K}(x)=\frac{x-\Theta_{K}(x)}{R_{K}(x)}
$$


It is natural to set $\nabla_{K}(x)=0$ for $x \in K$. For $x \in \mathbb{R}^{n} \backslash K$, one has the following relation [22]:

$$
\nabla_{K}(x)^{2}=1-\frac{\mathcal{F}_{K}(x)^{2}}{R_{K}(x)^{2}} .
$$

Equivalently, $\left\|\nabla_{K}(x)\right\|$ is the cosine of the (half) angle of the smallest cone with apex $x$ that contains $\Gamma_{K}(x)$. The map $x \mapsto\left\|\nabla_{K}(x)\right\|$ is lower semicontinuous [22], which means that for all $x_{0}, \liminf _{x \rightarrow x_{0}}\left\|\nabla_{K}(x)\right\| \geq\left\|\nabla_{K}\left(x_{0}\right)\right\|$. Although $\nabla_{K}$ is not continuous, it is shown in [22] that Euler schemes using $\nabla_{K}$ converge uniformly, as the integration step decreases, toward a continuous flow $\mathfrak{C}: \mathbb{R}^{+} \times \mathbb{R}^{n} \rightarrow \mathbb{R}^{n}$. The integral line of this flow starting at a point $x \in \mathbb{R}^{n}$ can be parameterized by arc length $s \mapsto \mathfrak{C}(t(s), x)$. It is possible to express the value of $R_{K}$ at the point $\mathfrak{C}(t(l), x)$ by integration along the integral line with length $l$ downstream the point $x$ :

$$
R_{K}(\mathfrak{C}(t(l), x))=R_{K}(x)+\int_{0}^{l}\left\|\nabla_{K}(\mathfrak{C}(t(s), x))\right\| d s .
$$

It is proven in [22] that the functions $\mathcal{F}_{K}$ and $R_{K}$ are increasing along the trajectories of the flow. In the particular case where $K$ is a finite set, various notions of flows related to this one have been independently introduced by Edelsbrunner [16], Giesen and al. [20], and R. Chaine [4] using Voronoï diagrams and Delaunay triangulations.

\subsection{Critical Point Theory for Distance Functions}

The critical points of $R_{K}$ are defined as the points $x$ for which $\nabla_{K}(x)=0$. Equivalently, a point $x$ is a critical point if and only if it lies in the convex hull of $\Gamma_{K}(x)$. When $K$ is finite, this last definition means that critical points are precisely the intersections of Delaunay $k$-dimensional simplices with their dual $(n-k)$-dimensional Voronoï facets [20]. Note that this notion of critical point is the same as the one considered in the setting of nonsmooth analysis [10] and Riemannian geometry [9, 21].

The topology of the offsets $K^{\alpha}$ of a compact set $K$ are closely related to the critical values of $R_{K}$ (i.e., the values of its distance function at critical points). The weak feature size of $K$, or wfs $(K)$, is defined as the infimum of the positive critical values of $R_{K}$. Equivalently it is the minimum distance between $K$ and the set of critical points of $R_{K}$. Notice that wfs $(K)$ may be equal to zero. The following result from [6] shows that wfs $(K)$ may be viewed as the "minimum size of the topological features" of the set $K$ :

Lemma 2.1 If $0<\alpha, \beta<\operatorname{wfs}(K)$, then $K^{\alpha}$ and $K^{\beta}$ are homeomorphic and even isotopic. The same holds for the complements of $K^{\alpha}$ and $K^{\beta}$.

Roughly speaking, two subspaces of $\mathbb{R}^{n}$ are isotopic if they can be deformed one into each other without tearing or self-intersection. For example, a circle and a trefoil knot are homeomorphic but not isotopic. 


\subsection{A Topological Approximation Result}

It has been shown in [6] that compact sets with weak feature size larger than a given constant satisfy a topological stability property. This property is defined in terms of homotopy type, which we now recall. Given two topological spaces $X$ and $Y$, two maps $f: X \rightarrow Y$ and $g: X \rightarrow Y$ are said to be homotopic if there is a continuous map $H:[0,1] \times X \rightarrow Y$ such that $\forall x \in X, H(0, x)=f(x)$ and $H(1, x)=g(x) . X$ and $Y$ are said to be homotopy equivalent if there are continuous maps $f: X \rightarrow Y$ and $g: Y \rightarrow X$ such that $g \circ f$ is homotopic to the identity map of $X$ and $f \circ g$ is homotopic to the identity map of $Y$. An equivalence class for homotopy equivalence is called a homotopy type. If two spaces $X$ and $Y$ are homeomorphic, then they are homotopy equivalent. In general, the converse is not true. For instance, a square and an annulus have the same homotopy type, though they are not homeomorphic. However, homotopy equivalence between topological spaces implies a one-to-one correspondance between connected components, cycles, holes, tunnels, cavities, or higher-dimensional topological features of the two sets. More precisely, if $X$ and $Y$ have same homotopy type, then their homotopy and homology groups are isomorphic.

The result of [6] we mentioned above is the following:

Theorem 2.2 Let $K$ and $K^{\prime}$ be compact subsets of $\mathbb{R}^{n}$, and let $\varepsilon$ be such that $\operatorname{wfs}(K)>2 \epsilon, \operatorname{wfs}\left(K^{\prime}\right)>2 \varepsilon$, and $d_{H}\left(K, K^{\prime}\right)<\varepsilon$. One has:

(i) $\mathbb{R}^{n} \backslash K$ and $\mathbb{R}^{n} \backslash K^{\prime}$ have the same homotopy type.

(ii) If $0<\alpha \leq 2 \varepsilon$, then $K^{\alpha}$ and $K^{\prime \alpha}$ have the same homotopy type.

We note that, in general, $\alpha$ cannot be set to 0 in the theorem. An example, given in [6], consists of a planar closed curve containing oscillations similar to the ones of the graph of $x \mapsto \sin (1 / x)$ near 0 . Because of these oscillations, the curve is simply connected. However any sufficiently small offset of the curve has a nontrivial fundamental group and hence a different homotopy type as the curve itself.

\section{Stability of Critical Points}

In this section, we give quantitative estimates of how much objects such as medial axes and critical points of the distance function to a compact set are affected by Hausdorff perturbation of the compact set.

\section{$3.1 \mu$-critical Points}

Let us first extend the notion of critical point of a distance function through the following definition:

Definition 3.1 ( $\mu$-critical point) A $\mu$-critical point of the compact set $K$ is a point at which the norm of the gradient $\nabla_{K}$ does not exceed $\mu$. 
Fig. 2 Proof of Lemma 3.2

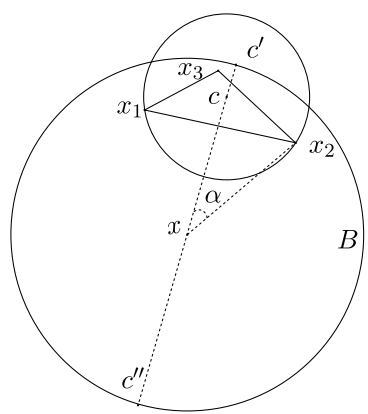

In particular, 0-critical points are exactly the critical points of the distance function to $K$. Also, a point is $\mu$-critical with $\mu<1$ if and only if it belongs to the medial axis of $\mathbb{R}^{n} \backslash K$. The concept of $\mu$-critical points is closely related to the $\theta$-medial axis introduced recently in $[14,19]$. The $\theta$-medial axis of a compact set $K$ is the set of points $p$ having at least two closest points $q$ and $r$ on $K$ such that the angle between $\overrightarrow{p q}$ and $\overrightarrow{p r}$ is at least $\theta$. Hence, a point having exactly two closest points on $K$ is in the $\theta$-medial axis if and only if it is $\cos (\theta / 2)$-critical. However, there is no such relationship between the two concepts for points having at least three closest points on $K$.

As the following lemma shows, the distance function to a compact set cannot grow too fast around a $\mu$-critical point:

Lemma 3.2 Let $K \subset \mathbb{R}^{n}$ be a compact set and $x$ one of its $\mu$-critical points. For any $y \in \mathbb{R}^{n}$, we have:

$$
R_{K}(y)^{2} \leq R_{K}(x)^{2}+2 \mu R_{K}(x)\|x-y\|+\|x-y\|^{2} .
$$

Proof Let $\Gamma$ be the set of points closest to $x$ on $K$, and let $B$ be the sphere centered at $x$ and containing $\Gamma$. Let also $c$ be the center of the minimal enclosing ball of $\Gamma$, and $\alpha=\cos ^{-1}(\mu)$ (see Fig. 2, where $\Gamma=\left\{x_{1}, x_{2}, x_{3}\right\}$ ).

We now claim that $\overrightarrow{x y}$ makes an angle at most $\pi-\alpha$ with a vector $\overrightarrow{x x^{\prime}}$ for some $x^{\prime} \in \Gamma$. Without loss of generality, we can assume that $y$ belongs to $B$. First assume that $x$ belongs to the convex hull of $\Gamma$, i.e., $\alpha$ equals $\pi / 2$. Equivalently, $\Gamma$ is not contained in any hemisphere, meaning that the property is satisfied for all $y$. If this is not the case, $\Gamma$ is contained in some hemisphere. We then have that the center of the minimal enclosing geodesic disk of $\Gamma$ is the point $c^{\prime}$ where the ray $[x c$ ) pierces $B$. By considering the complement of the disk, we get that the geodesic furthest point to $\Gamma$ on $B$ is the antipode $c^{\prime \prime}$ of the center of its minimal enclosing disk $c^{\prime}$. The desired inequality on angles is clearly satisfied when $y$ equals $c^{\prime \prime}$, since the angle $\alpha^{\prime}$ between $\overrightarrow{x c^{\prime}}$ and the vector $\overrightarrow{x x^{\prime}}$ equals $\alpha$ whenever $x^{\prime}$ belongs to the minimal enclosing sphere of $\Gamma$. However, since $c^{\prime \prime}$ is the point farthest to $\Gamma$ on $B$, this is the worst case for $y$, which concludes the proof of our angle inequality.

To conclude the proof of the lemma, it is sufficient to notice that $R_{K}(y) \leq\left\|y-x^{\prime}\right\|$ and expand $\left\|y-x^{\prime}\right\|^{2}=\left\langle(y-x)+\left(x-x^{\prime}\right),(y-x)+\left(x-x^{\prime}\right)\right\rangle$. 
The same lemma already appeared in [22] with a different proof. We include it here for the sake of completeness.

3.2 Behavior under Hausdorff Approximation

We now use Lemma 3.2 to understand how the $\mu$-critical points of a compact set $K$ change when $K$ is replaced by a compact set $K^{\prime}$ having a small Hausdorff distance with $K$. Let us first state the following:

Lemma 3.3 Let $K$ and $K^{\prime}$ be two compact subsets of $\mathbb{R}^{n}$ and $d_{H}\left(K, K^{\prime}\right) \leq \varepsilon$. For any $\mu$-critical point $x$ of $K$ and any $\rho>0$, there is a $\mu^{\prime}$-critical point of $K^{\prime}$ at distance at most $\rho$ from $x$, with

$$
\mu^{\prime} \leq \frac{\rho}{2 R_{K}(x)}+2 \frac{\varepsilon}{\rho}+\mu .
$$

Proof Let us consider an integral line $C$ of the vector field $\nabla_{K^{\prime}}$ parameterized by arc length and starting at $x$. If $C$ reaches a critical point of $K^{\prime}$ before length $\rho$, the lemma holds. Assume this is not the case. Letting $y=C(\rho)$, we have:

$$
R^{\prime}(y)-R^{\prime}(x)=\int_{0}^{\rho}\left\|\nabla_{K^{\prime}}(C(s))\right\| d s,
$$

where $R$ (resp. $R^{\prime}$ ) denotes $R_{K}$ (resp. $R_{K^{\prime}}$ ). Therefore, since $\left\|\nabla_{K}\right\|$ is lower semicontinuous, there must exist a point $p$ on the curve such that:

$$
\left\|\nabla_{K^{\prime}}(p)\right\| \leq \frac{R^{\prime}(y)-R^{\prime}(x)}{\rho} .
$$

Note that $\|p-x\| \leq \rho$. Now Lemma 3.2 applied to $x, y$, and $K$ reads:

$$
R(y) \leq \sqrt{R(x)^{2}+2 \mu R(x)\|x-y\|+\|x-y\|^{2}} .
$$

Also, since $\varepsilon=d_{H}\left(K, K^{\prime}\right)$, we have that for all $z \in \mathbb{R}^{n},\left|R(z)-R^{\prime}(z)\right| \leq \varepsilon$. Hence:

$$
\begin{aligned}
R^{\prime}(y)-R^{\prime}(x) \leq & \sqrt{R(x)^{2}+2 \mu R(x)\|x-y\|+\|x-y\|^{2}} \\
& -R(x)+2 \varepsilon \\
\leq & R(x)\left[\sqrt{\left.1+\frac{2 \mu\|x-y\|}{R(x)}+\frac{\|x-y\|^{2}}{R(x)^{2}}-1\right]}\right. \\
& +2 \varepsilon \\
\leq & \mu\|x-y\|+\frac{\|x-y\|^{2}}{2 R(x)}+2 \varepsilon
\end{aligned}
$$

the last inequality coming from the fact that the square root function is concave. Noticing that $\|x-y\| \leq \rho$, dividing by $\rho$, and applying (1) shows that $p$ satisfies the desired requirements. 
As a function of $\rho$, the upper bound on $\mu^{\prime}$ obtained in the previous lemma attains its minimum at $\rho=2 \sqrt{\varepsilon R_{K}(x)}$ and equals $2 \sqrt{\varepsilon / R_{K}(x)}+\mu$ at that point. We thus have:

Theorem 3.4 (Critical point stability theorem) Let $K$ and $K^{\prime}$ be two compact subsets of $\mathbb{R}^{n}$ and $d_{H}\left(K, K^{\prime}\right) \leq \varepsilon$. For any $\mu$-critical point $x$ of $K$, there is a $\left(2 \sqrt{\varepsilon / R_{K}(x)}+\right.$ $\mu)$-critical point of $K^{\prime}$ at distance at most $2 \sqrt{\varepsilon R_{K}(x)}$ from $x$.

In what follows, we will need the fact that this critical point $p$ of $K^{\prime}$ can be chosen such that $R_{K^{\prime}}(p) \geq R_{K^{\prime}}(x)$, which is obvious from the proof of Lemma 3.3.

To conclude this section, we show that the previous theorem implies a convergence result for $\mu$-medial axes. The $\mu$-medial axis of a compact set $K$, denoted by $\mathcal{M}_{\mu}(K)$, is the set of points $x \notin K$ such that $\left\|\nabla_{K}(x)\right\| \leq \mu$. Note that the $\mu$ medial axis is different from the $\lambda$-medial axis introduced in [5]. Since $\left\|\nabla_{K}\right\|$ is lower semicontinuous, the set $\mathcal{M}_{\mu}(K) \cup K$ is compact for any $\mu \in(0,1)$. So the map $\mu \rightarrow \mathcal{M}_{\mu}(K) \cup K$ with values in the metric space (for Hausdorff distance) of compact sets of $\mathbb{R}^{n}$ is continuous at $\mu$ if and only if for any sequence $\mu_{n} \rightarrow \mu$, $d_{H}\left(\mathcal{M}_{\mu_{n}}(K) \cup K, \mathcal{M}_{\mu}(K) \cup K\right) \rightarrow 0$ as $n \rightarrow \infty$.

Theorem 3.5 ( $\mu$-medial axis convergence theorem) Let $K$ be a compact subset of $\mathbb{R}^{n}$, and $\left(K_{i}\right)$ be a sequence of compact sets converging to $K$ for the Hausdorff distance. If $\mu \in(0,1)$ is a continuity point of the map $\mu \rightarrow \mathcal{M}_{\mu}(K) \cup K$, then $\mathcal{M}_{\mu}\left(K_{i}\right) \cup K_{i}$ converges to $\mathcal{M}_{\mu}(K) \cup K$ for the Hausdorff distance.

Related results were obtained in [5] for a different filtration of the medial axis.

Proof To simplify the notation, we denote by $\tilde{\mathcal{M}}_{\mu}(K)=\mathcal{M}_{\mu}(K) \cup K$ the union of a compact with its $\mu$-medial axis. Since

$$
\left\|\nabla_{K}(x)\right\|^{2}=1-\frac{\mathcal{F}_{K}(x)^{2}}{R_{K}(x)^{2}}
$$

and $\mathcal{F}_{K}(x)$ is bounded by the diameter of $K$, it follows that $\mathcal{M}_{\mu}(K)$ is contained in any ball with center in $K$ and radius $D>\operatorname{diam}(K)$ such that

$$
1-\frac{\operatorname{diam}(K)^{2}}{(D-\operatorname{diam}(K))^{2}}>\mu^{2} .
$$

It follows from the critical point stability Theorem 3.4 that if $K^{\prime}$ is a compact such that $d_{H}\left(K, K^{\prime}\right)<\varepsilon$, then

$$
\tilde{\mathcal{M}}_{\mu}(K) \subset \tilde{\mathcal{M}}_{\mu+2 \varepsilon^{1 / 4}}\left(K^{\prime}\right)^{\varepsilon+2 \sqrt{D \varepsilon}}
$$

assuming that $\varepsilon<\operatorname{diam}(K)$. To prove this claim we consider two cases: given $x \in$ $\tilde{\mathcal{M}}_{\mu}(K)$, if $R_{K}(x)<\sqrt{\varepsilon}$, then $d\left(x, K^{\prime}\right)<\varepsilon+\sqrt{\varepsilon}<\varepsilon+2 \sqrt{D \varepsilon}$, and so the claim is true. Otherwise, we apply the critical point stability theorem to $x$, which directly yields the result. 
Using the previous claim twice, we obtain

$$
\tilde{\mathcal{M}}_{\mu-2 \varepsilon^{1 / 4}}(K) \subset \tilde{\mathcal{M}}_{\mu}\left(K^{\prime}\right)^{\varepsilon+2 \sqrt{D \varepsilon}} \subset \tilde{\mathcal{M}}_{\mu+2 \varepsilon^{1 / 4}}(K)^{2 \varepsilon+4 \sqrt{D \varepsilon}} .
$$

Since $\mu \rightarrow \tilde{\mathcal{M}}_{\mu}(K)$ is continuous at $\mu$, one has

$$
\begin{gathered}
d_{H}\left(\tilde{\mathcal{M}}_{\mu-2 \varepsilon^{1 / 4}}(K), \tilde{\mathcal{M}}_{\mu}(K)\right) \rightarrow 0 \text { and } \\
d_{H}\left(\tilde{\mathcal{M}}_{\mu+2 \varepsilon^{1 / 4}}(K)^{2 \varepsilon+4 \sqrt{D \varepsilon}}, \tilde{\mathcal{M}}_{\mu}(K)\right) \rightarrow 0
\end{gathered}
$$

as $\varepsilon \rightarrow 0$. Replacing $K^{\prime}$ by the $K_{i}$ 's concludes the proof of the theorem.

The convergence of the $\mu$-medial axis is guaranteed only for values $\mu$ at which the map $\mu \rightarrow \mathcal{M}_{\mu}(K) \cup K$ is continuous. The following result shows that the set of discontinuity points is finite for a large class of compact sets.

Theorem 3.6 Let $K$ be a subanalytic compact subset of $\mathbb{R}^{n}$. For all but a finite number of values $\mu, \mu \rightarrow \mathcal{M}_{\mu}(K) \cup K$ is continuous.

Subanalytic sets are a large class of subsets that includes, for instance, finite sets, semi-algebraic sets, and sets defined by analytic equalities and inequalities. For precise definitions and references on subanalytic geometry, see [8] for instance.

Proof The proof of the theorem, which is based upon classical tools in subanalytic geometry that are rather different than the ones used in this paper, is omitted. It is almost the same as the proof of Theorem 6 in [5].

\section{A Sampling Theory for Compact Sets}

Based on the results obtained in the previous sections, we are now able to formulate a theoretical framework for inferring the topology and geometry of a large class of shapes from Hausdorff approximations.

\subsection{The Critical Function of a Compact Set}

We first introduce a function that one can associate with any compact set and that encodes all the " $\mu$-critical values" of the distance function to that compact set.

Definition 4.1 (Critical function) Given a compact set $K \subset \mathbb{R}^{n}$, its critical function $\chi_{K}:(0,+\infty) \rightarrow \mathbb{R}_{+}$is the real function defined by

$$
\chi_{K}(d)=\inf _{R_{K}^{-1}(d)}\left\|\nabla_{K}\right\|
$$


We note that the infimum can be replaced by the minimum since $\left\|\nabla_{K}\right\|$ is lower semi-continuous and $R_{K}^{-1}(d)$ is compact. It also results from the compactness of $R_{K}^{-1}(d)$ that $d \mapsto \chi_{K}(d)$ is lower semi-continuous. As we will see, whether a compact set is a Hausdorff approximation of a "simple" compact set or not can be read from its critical function, which is the main motivation for introducing this concept. To back up this claim, we will need the following technical result:

Theorem 4.2 (Critical function stability theorem) Let $K$ and $K^{\prime}$ be two compact subsets of $\mathbb{R}^{n}$ and $d_{H}\left(K, K^{\prime}\right) \leq \varepsilon$. For all $d \geq 0$, we have:

$$
\inf \left\{\chi_{K^{\prime}}(u) \mid u \in I(d, \varepsilon)\right\} \leq \chi_{K}(d)+2 \sqrt{\frac{\varepsilon}{d}},
$$

where $I(d, \varepsilon)=\left[d-\varepsilon, d+2 \chi_{K}(d) \sqrt{\varepsilon d}+3 \varepsilon\right]$.

Proof Let $d \geq 0$ and $x$ be a point in $R_{K}^{-1}(d)$ such that $\left\|\nabla_{K}(x)\right\|=\chi_{K}(d)$. Denoting $\chi_{K}(d)$ by $\chi$ to simplify the notation, we know by the Critical Point Stability Theorem that there is a point $p$ that is $(2 \sqrt{\varepsilon / d}+\chi)$-critical for $K^{\prime}$ and that lies at distance at most $2 \sqrt{\varepsilon d}$ from $x$. Applying Lemma 3.2 to $K$ and the points $x$ and $p$, we get:

$$
\begin{aligned}
R_{K}(p) & \leq \sqrt{d^{2}+4 \chi d \sqrt{\varepsilon d}+4 \varepsilon d} \\
& \leq d \sqrt{1+4 \chi \sqrt{\varepsilon / d}+4 \varepsilon / d} \\
& \leq d+2 \chi \sqrt{\varepsilon d}+2 \varepsilon .
\end{aligned}
$$

Recalling that $p$ can be chosen such that $R_{K^{\prime}}(p) \geq R_{K^{\prime}}(x)$ and that $\mid R_{K^{\prime}}(p)-$ $R_{K}(p) \mid \leq \varepsilon$ concludes the proof.

In particular, this stability property implies that the epigraph of $\chi_{K}$ converges to the one of $\chi_{K^{\prime}}$ for the Hausdorff distance as $\varepsilon$ goes to $0 .^{1}$

From a practical point of view, we note that it is feasible to compute the critical function of a point cloud in $\mathbb{R}^{3}$. Indeed, it follows from the definition that this function is the minimum of functions associated with each Voronoi cell. For the Voronoi cell $V_{\tau}$ dual to Delaunay simplex $\tau$, the corresponding function is $f_{\tau}: R_{K}\left(V_{\tau}\right) \rightarrow \mathbb{R}$ defined by:

$$
f_{\tau}(d)=\sqrt{1-\frac{\mathcal{F}(\tau)^{2}}{d^{2}}} .
$$

Hence, after appropriate transformations, computing the critical function boils down to computing the upper envelope of the horizontal line segments $R_{K}\left(V_{\tau}\right) \times\{\mathcal{F}(\tau)\} \subset$ $\mathbb{R}^{2}$, where $\tau$ ranges over the Delaunay triangulation of the point cloud. Figure 3 shows the critical function obtained by this process in the case of a sampling of a square

\footnotetext{
${ }^{1}$ The epigraph of a real function $f$ is the set of points $(x, y)$ such that $f(x) \leq y$.
} 
Fig. 3 Critical function of a square embedded in $\mathbb{R}^{3}$ with side length 50 (top), and of a sampling of that square (bottom)
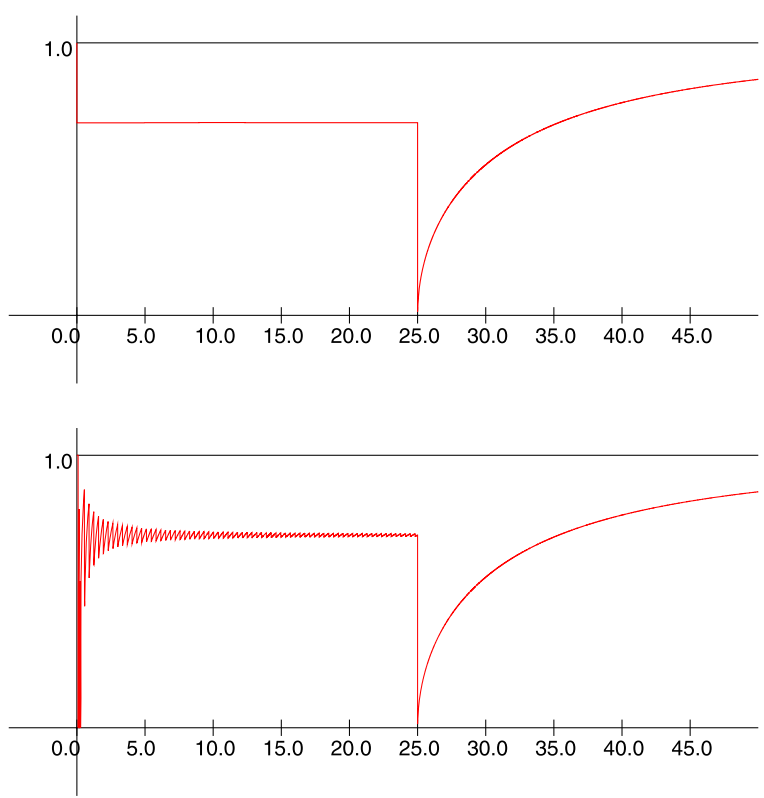

with sidelength 50 lying in $\mathbb{R}^{3}$. The Hausdorff distance between the sampling and the square is 0.25 . The critical function of the square equals $\sqrt{2} / 2$ for $0<d<25$, 0 for $d=25$, and $\sqrt{1-25^{2} / d^{2}}$ for $d>25$. The critical function of the sampling is very close to the one the square, except for small $d$, which is consistent with the theorem.

\section{$4.2 \mu$-reach}

Using the critical function, we can define a parameterized notion of "feature size" that interpolates between the minimum local feature size and the weak feature size:

Definition 4.3 ( $\mu$-reach) The $\mu$-reach $r_{\mu}(K)$ of a compact set $K \subset \mathbb{R}^{n}$ is defined by

$$
r_{\mu}(K)=\inf \left\{d \mid \chi_{K}(d)<\mu\right\}
$$

Equivalently, $r_{\mu}(K)$ is the minimum distance between a point in $K$ and a point in the closure of $\mathcal{M}_{\mu}(K)$.

We have that $r_{1}(K)$ coincides with the minimum over $K$ of the local feature size function $[1,2]$. Note that this number is also the same as the reach of $K$, which was introduced by Federer [18] in the context of the study of curvature measures. As $\mu$ increases, $r_{\mu}(K)$ decreases and $\lim _{\mu \rightarrow 0^{+}} r_{\mu}(K) \leq \mathrm{wfs}(K)$. The inequality can be strict: for instance, if $K$ is the union of two tangent disks, then the $\mu$-reach of $K$ is 0 for all $\mu>0$, whereas $\mathrm{wfs}(K)=+\infty$. However, if the limit is positive, then equality holds. In any case, it can be proved using the lower semi-continuity of the critical function that $\lim _{\varepsilon \rightarrow 0^{+}}\left(\lim _{\mu \rightarrow 0^{+}} r_{\mu}\left(K^{\varepsilon}\right)\right)=\operatorname{wfs}(K)$. 
The advantage of this notion over the reach is that it is nonzero-for well-chosen $\mu$-for a large class of objects. For example, as is well known, piecewise-linear surfaces have zero reach since their sharp edges are in the closure of the medial axis of their complement. However, if $K$ is a piecewise-linear surface, then there is a maximum real number $\lambda$ such that no ball with radius less than $\lambda$ intersects at least two nonadjacent faces. This number was introduced by Ruppert [26] in the context of mesh generation. Now for a vertex of $K$, we can consider the angle of the minimal enclosing cone of the normals of the facets incident to the vertex. Calling $\theta$ the minimum of these angles over all vertices and letting $\mu=\cos (\theta / 2)$, we have that $r_{\mu} \geq \lambda$.

The weak feature size is also nonzero for a large class of shapes. However, we will see that the weak feature size is not sufficient for our purpose. The main reason for this is given in the next paragraph.

\subsection{Separation of Critical Values}

The fact that a compact set is close to a compact set with positive $\mu$-reach implies restrictions on the possible locations of the critical values of its distance function, as we now show. A result similar to ours was proven by Dey et al. [15] recently but in the special case of closed surfaces smoothly embedded in $\mathbb{R}^{3}$ and sampled without noise.

Theorem 4.4 (Critical values separation theorem) Let $K$ and $K^{\prime}$ be two compact subsets of $\mathbb{R}^{n}$, $\varepsilon$ be the Hausdorf distance between $K$ and $K^{\prime}$, and $\mu$ be a nonnegative number. The distance function $R_{K}$ has no critical values in the interval ] $4 \varepsilon / \mu^{2}, r_{\mu}\left(K^{\prime}\right)-3 \varepsilon\left[\right.$. Besides, for any $\mu^{\prime}<\mu, \chi_{K}$ is larger than $\mu^{\prime}$ on the interval

$$
] \frac{4 \varepsilon}{\left(\mu-\mu^{\prime}\right)^{2}}, r_{\mu}\left(K^{\prime}\right)-3 \sqrt{\varepsilon r_{\mu}\left(K^{\prime}\right)}[.
$$

Before proving the theorem, we note that taking $\mu$ too small does not give any information on the critical values, since the lower bound then exceeds the upper bound. This is why it is preferable to work with the $\mu$-reach than with the weak feature size.

Proof Let us prove the first part of the theorem. Assume that $d$ is a critical value of $R_{K}$, i.e., $\chi_{K}(d)=0$. By the critical function stability theorem, we have that

$$
\inf \left\{\chi_{K^{\prime}}(u) \mid u \in[d-\varepsilon, d+3 \varepsilon]\right\} \leq 2 \sqrt{\frac{\varepsilon}{d}} .
$$

So if $d<r_{\mu}\left(K^{\prime}\right)-3 \varepsilon$, this implies that $\mu \leq 2 \sqrt{\varepsilon / d}$, which implies that $d$ cannot belong to the desired interval. Now for the second part of the theorem, we assume that $d$ is a $\mu^{\prime}$-critical value of $R_{K}$ and apply the same theorem again. This time we get that if

$$
d+2 \chi_{K}(d) \sqrt{\varepsilon d}+3 \varepsilon<r_{\mu}\left(K^{\prime}\right)
$$

then $\mu \leq \mu^{\prime}+2 \sqrt{\varepsilon / d}$, which is precisely the lower bound of the desired interval. Now the former condition can be successively strenghtened as follows:

$$
d+2 \sqrt{\varepsilon d}+3 \varepsilon<r_{\mu}\left(K^{\prime}\right),
$$


Fig. 4 The separation bounds on critical values are almost tight

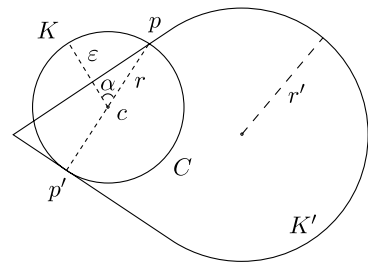

$$
\begin{aligned}
(\sqrt{d}+\sqrt{\varepsilon})^{2} & <r_{\mu}\left(K^{\prime}\right)-2 \varepsilon, \\
d & <\left(\sqrt{r_{\mu}\left(K^{\prime}\right)-2 \varepsilon}-\sqrt{\varepsilon}\right)^{2}, \\
d & <r_{\mu}\left(K^{\prime}\right)-\varepsilon-2 \sqrt{\varepsilon\left(r_{\mu}\left(K^{\prime}\right)-2 \varepsilon\right),} \\
d & <r_{\mu}\left(K^{\prime}\right)-\varepsilon-2 \sqrt{\varepsilon r_{\mu}\left(K^{\prime}\right)}, \\
d & <r_{\mu}\left(K^{\prime}\right)-3 \sqrt{\varepsilon r_{\mu}\left(K^{\prime}\right)},
\end{aligned}
$$

where the latter inequality assumes $\varepsilon \leq r_{\mu}\left(K^{\prime}\right)$. Since the theorem is also true (with a void conclusion) when this condition is not satisfied, this achieves the proof.

The bounds given in the first part of the theorem are actually tight up to a multiplicative constant. An example is shown in Fig. 4, where $K^{\prime}$ is a union of a circle arc and two segments. The circle arc meets the two segments tangentially, and these form an angle $\alpha$ equal to $2 \sin ^{-1}(\mu)$ for some $\mu>0$. The critical function $\chi_{K^{\prime}}(d)$ equals $\mu$ for $0<d<r^{\prime}$, where $r^{\prime}$ is the radius of the circle arc. Since the center of the circle is critical, we have $\chi_{K^{\prime}}\left(r^{\prime}\right)=0$, so $r_{\mu}\left(K^{\prime}\right)=r^{\prime}$. For the tightness of the upper bound, just take for $K$ the set of points at distance at most $\varepsilon$ from $K^{\prime}$, assuming that $\varepsilon<r^{\prime}$. Then we have that the center of the circle is a critical point of the distance function to $K$, with $r^{\prime}-\varepsilon$ as corresponding critical value. For the lower bound, consider a point $p$ on one of the segments, and let $p^{\prime}$ be its closest point on the other segment (here we assume that $\alpha<\pi / 2$ ). Let $C$ be the circle with diameter $p p^{\prime}$. To obtain $K$ from $K^{\prime}$, replace the part of $K^{\prime}$ enclosed by $C$ by the circle arc of $C$ bulging out of $K^{\prime}$. The center $c$ of $C$ is a critical point of $R_{K}$. Calculations shows that the ratio between $\varepsilon=d_{H}\left(K, K^{\prime}\right)$ and the corresponding critical value $r$ is $1-\cos (\alpha)$. Since $1-\cos (\alpha)=\Theta\left(\sin ^{2}(\alpha)\right)=\Theta\left(\mu^{2}\right)$, the lower bound is also tight up to a multiplicative constant.

\subsection{Reconstruction by Offsets}

We now formulate a sampling condition inspired by the work of Amenta et al. [1, 2, 23]:

Definition $4.5((\kappa, \mu)$-approximation) Given two nonnegative real numbers $\kappa$ and $\mu$, we say that a compact $K \subset \mathbb{R}^{n}$ is a $(\kappa, \mu)$-approximation of a compact $K^{\prime} \subset \mathbb{R}^{n}$ if the Hausdorff distance between $K$ and $K^{\prime}$ does not exceed $\kappa$ times the $\mu$-reach of $K^{\prime}$. 
Fig. 5 Distance function to a sampling of an equilateral triangle. If the offset parameter is appropriately chosen, then the offset of the sampling (its boundary is shown in bold) is homotopy equivalent to the triangle

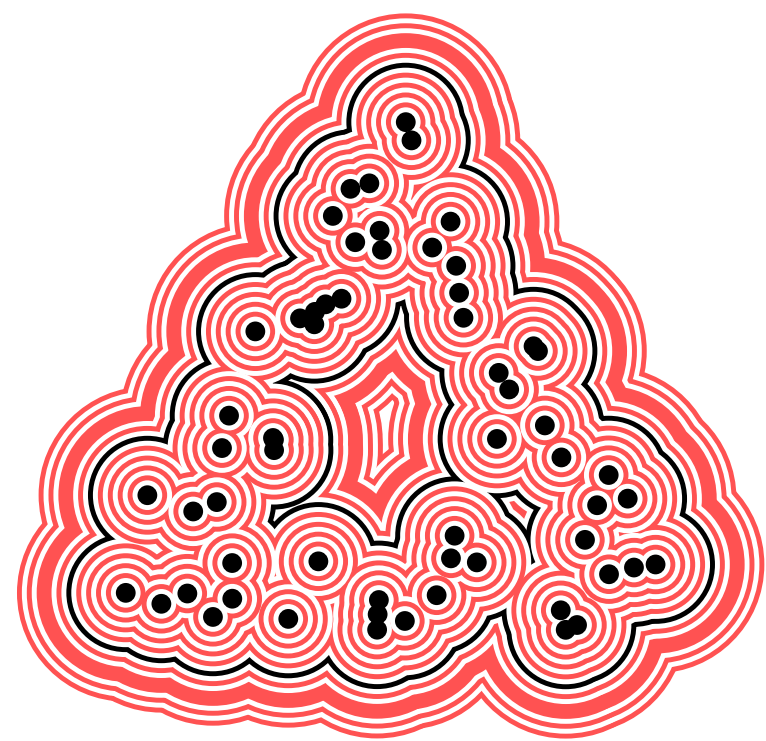

In particular, if $K^{\prime}$ is an embedded surface in $\mathbb{R}^{3}$, finite $(\kappa, \mu)$-approximations are related to noisy $(\varepsilon, \kappa)$-samples introduced in [12], and finite $(\kappa, 1)$-approximations are related to $\varepsilon$-samples of $K$ (with $\varepsilon=\kappa$ ) as defined in [23]. One difference is that the points in an $\varepsilon$-sample are assumed to lie on the surface, whereas $(\kappa, \mu)$ approximations can be noisy. The other difference is that $\varepsilon$-samples are allowed to be less dense in areas where the surface is not too curved and where the object bounded by the surface is not too "thin." This local adaptivity of $\varepsilon$-samples is a nice feature, but unfortunately nonsmooth shapes as simple as polyhedra do not admit-finite- $\varepsilon$ samples, even when the sharp edges are arbitrarily flat. In contrast, polyhedra admit finite $(\kappa, \mu)$-approximations, since they have positive $\mu$-reach for suitable $\mu$ as explained before.

An important property of $(\kappa, \mu)$-approximations of a compact set is that they allow one to reconstruct the compact set in a topologically correct way using simple offsets (see Fig. 5).

Theorem 4.6 (Reconstruction theorem) Let $K \subset \mathbb{R}^{n}$ be $a(\kappa, \mu)$-approximation of a compact set $K^{\prime}$. If

$$
\kappa<\frac{\mu^{2}}{5 \mu^{2}+12}
$$

then the complement of $K^{\alpha}$ is homotopy equivalent to the complement of $K^{\prime}$, and $K^{\alpha}$ is homotopy equivalent to $K^{\prime \eta}$ for sufficiently small $\eta$, provided that

$$
\frac{4 d_{H}\left(K, K^{\prime}\right)}{\mu^{2}} \leq \alpha<r_{\mu}\left(K^{\prime}\right)-3 d_{H}\left(K, K^{\prime}\right) .
$$


Proof By the Separation of Critical Values Theorem, we know that $R_{K}$ has no critical values in the interval $] 4 \varepsilon / \mu^{2}, r_{\mu}\left(K^{\prime}\right)-3 \varepsilon$ [, where $\varepsilon$ denotes $d_{H}\left(K, K^{\prime}\right)$. Hence by Lemma 2.1 the (complements of) $K^{\alpha}$ all have the same homotopy type when $\alpha$ belongs to this interval (including its lower bound). We may thus prove the theorem for $\alpha=4 \varepsilon / \mu^{2}$. Since performing an offset on a compact set translates into translating its critical function to the left by the same value, we get that

$$
\operatorname{wfs}\left(K^{\alpha}\right) \geq r_{\mu}\left(K^{\prime}\right)-3 \varepsilon-\frac{4 \varepsilon}{\mu^{2}} .
$$

Also:

$$
d_{H}\left(K^{\alpha}, K^{\prime}\right) \leq \frac{4 \varepsilon}{\mu^{2}}+\varepsilon .
$$

By a result in [6], Theorem 1 recalled in Sect. 2, a sufficient condition for the conclusion of the theorem to be true is that

$$
d_{H}\left(K^{\alpha}, K^{\prime}\right)<\frac{1}{2} \min \left(\operatorname{wfs}\left(K^{\alpha}\right), \operatorname{wfs}\left(K^{\prime}\right)\right)
$$

The assumption on $\kappa$ is precisely such that this is the case.

When $K^{\prime}$ is a closed-unnecessarily smooth-connected surface embedded in $\mathbb{R}^{3}$, the conclusion of the Reconstruction theorem can be strengthened. Using results in [6], we have indeed that each boundary component of the offset of $K$ is isotopic to $K^{\prime}$. Besides, we note that a result similar to our Reconstruction Theorem has been obtained by Niyogi, Smale, and Weinberger [24]. The constants in this work are better than ours, ${ }^{2}$ but the setting is limited to smoothly embedded hypersurfaces. Also, when $K$ is finite, we may substitute the offset by the $\alpha$-shape [17] with the same value of the parameter, since the two are known to be homotopy equivalent [16].

\subsection{Parameter Selection}

The main shortcoming of the Reconstruction Theorem is that it does not give a way to find a suitable value for the offset parameter. From this perspective, a natural question is how can we tell whether a given compact set $K$ is a $(\kappa, \mu)$-approximation of some compact set $K^{\prime}$ ? We claim that this question can be answered to some extent by analyzing the critical function of $K$.

First, if $K$ is a $(\kappa, \mu)$-approximation of a compact set $K^{\prime}$, the Separation of Critical Values Theorem implies that for all $\mu^{\prime}>0$, the critical function $\chi_{K}$ is larger than $\mu^{\prime}$ on the interval

$$
] \frac{4 d_{H}\left(K, K^{\prime}\right)}{\left(\mu^{\prime}-\mu\right)^{2}}, r_{\mu}\left(K^{\prime}\right)(1-3 \sqrt{\kappa})[\text {. }
$$

\footnotetext{
${ }^{2}$ Approximately by a factor 3 .
} 
If we take, for instance, $\mu^{\prime}=\mu / 2$, the ratio between the bounds of this interval is at least

$$
\rho=\frac{(1-3 \sqrt{\kappa})}{16 \kappa} \mu^{2}
$$

Note that for small enough $\kappa$ and for fixed $\mu$, this ratio $\rho$ is proportional to $\kappa^{-1}$. Hence, the critical function of a $(\kappa, \mu)$-approximation with small $\kappa$ has to be larger than $\mu / 2$ on a "long" interval, more precisely on an interval whose ratio is at least $\rho$. This means that looking for all such intervals is a valid strategy for finding a super-set of the pairs $(\kappa, \mu)$ such that $K$ is a $(\kappa, \mu)$-approximation of some compact set, at least for small enough $\kappa$.

Conversely, the strategy we just described cannot yield "false positives," that is, sufficiently long intervals where the critical function is high, always witness the existence of a compact $K^{\prime}$ being $(\kappa, \mu)$-approximated by $K$. Indeed, if we find that $\chi_{K}$ is larger than $\mu$ on an interval of the form $] a, a \rho\left[\right.$, then $K$ is obviously a $\left((\rho-1)^{-1}, \mu\right)$ approximation of its $a$-offset. Hence, the lower bounds of these "long" intervals are convenient choices for the offset parameter.

We note that the critical function of a compact set can be larger than some constant $\mu$ on several disjoint intervals with arbitrarily large ratios. In this case, each one of these interval could correspond to a different homotopy type. Of course, if one has prior information on either $\kappa, \mu$, or the Hausdorff distance between $K$ and $K^{\prime}$, it is possible to restrict the set of possible homotopy types by narrowing the search for long intervals to the set of intervals satisfying the corresponding constraints.

This stands in sharp contrast with the sampling theory developed by Amenta et al. Indeed, a point set cannot be a $\kappa$-sampling of surfaces with different homotopy types for $\kappa<0.06$, since there is a provably correct algorithm that recovers the surface under this sampling condition [2]. However, this unicity property cannot hold if one allows noisy samples. For example, let $K_{1}$ be a noise free sampling of the unit sphere such that the Hausdorff distance between $K_{1}$ and the unit sphere is $\varepsilon$, and the distance between any sample and its closest sample is not smaller than $\varepsilon / 2$. Now let $K_{2}$ be the point cloud obtained from $K_{1}$ by replacing each sample $p$ by a copy of $K_{1}$ scaled by $\varepsilon^{2}$ and centered on $p$. Then we clearly have two plausible homotopy types for $K_{2}$, namely the one of the sphere or the one of the disjoint union of $\sharp K_{1}$ spheres. This construction can obviously be iterated to get any number of different homotopy types.

We end this section with a real world example. Figure 6 shows a sampled gearshift together with its critical function. Critical values appear in three ranges. Critical values induced by neighboring sample points lie in the interval $[0,2.5]$. Then between 9 and 10 , the neck of the model gets closed, which creates a void that fills in approximately at 20. We see that the critical function is high (larger than 0.4 ) on the interval $[2.5,9]$. This implies in particular that the sampling is a $(0.4,0.4)$-approximation of its 2.5-offset, which has the correct homotopy type, namely the one of a disk. Intervals included in $[10,20]$, which correspond to the homotopy type of a sphere, have smaller values of $\mu$ and smaller ratios. This is in accordance with the fact that the homotopy type of a sphere is visually less plausible than the one of a disk. 
Fig. 6 A sampled gearstick knob and its critical function
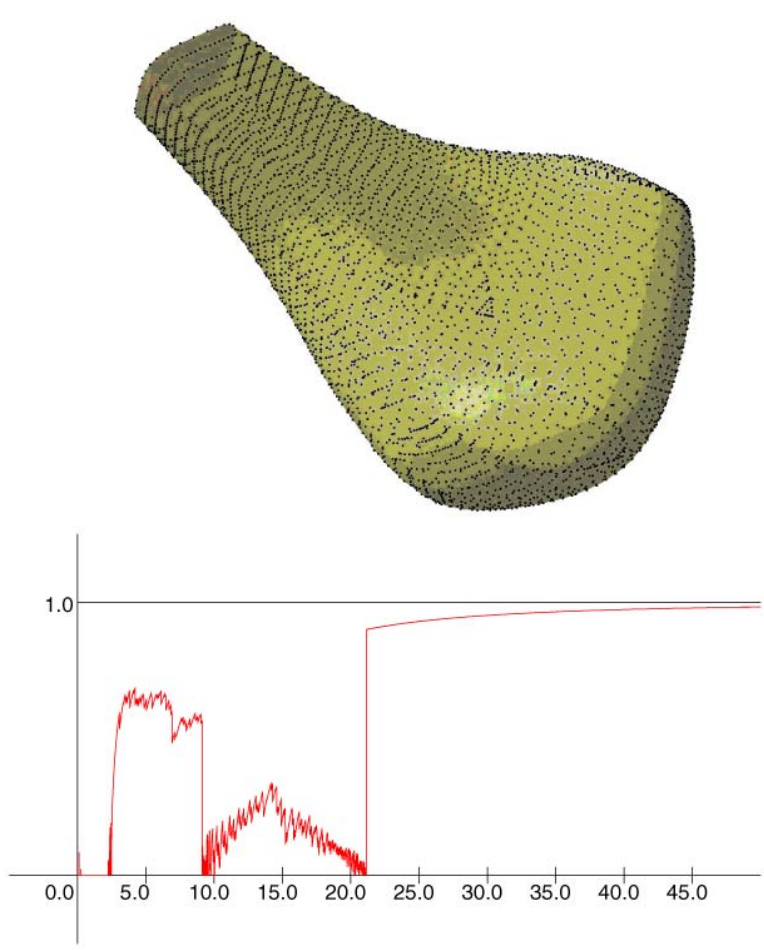

\section{Discussion}

Summary In this paper, we have formulated a parameterized set of sampling conditions under which the geometry and topology of a compact subset of Euclidean space can be recovered from an approximation of it. Also, these conditions allow one to determine which compact sets are well sampled by a given compact set, at least to some extent. Moreover, this task only requires to analyze the critical function of the given compact set, which is a real function defined on the set of positive real numbers. Our approach also yields new stability results for medial axes, critical points, and critical values of distance functions.

Future Work Several important questions remain wide open. First, do our sampling conditions allow one to recover differential information, for example, orientation of tangent spaces? A key question in this respect seems to be the stability of the gradient field of distance functions under Hausdorff perturbations. Second, the main shortcoming of our approach is that it assumes that the magnitude of the perturbation is uniform over the object, since we use Hausdorff distance. If this is not the case, it might be difficult to find a uniform offset parameter leading to a correct reconstruction. Is it possible to generalize our ideas to design a nonuniform sampling theory in the spirit of the one developed by Amenta and Bern? Finally, what can be said when the ambient metric space is not Euclidean? 


\section{References}

1. Amenta, N., Bern, M.: Surface reconstruction by Voronoi filtering. Discrete Comput. Geom. 22(4), 481-504 (1999)

2. Amenta, N., Choi, S., Dey, T., Leekha, N.: A simple algorithm for homeomorphic surface reconstruction. Int. J. Comput. Geom. Appl. 12(1, 2), 125-141 (2002)

3. Boissonnat, J.D., Oudot, S.: Provably good sampling and meshing of Lipschitz surfaces. In: Graphical Models (GMOD), vol. 67, pp. 405-451 (2005)

4. Chaine, R.: A geometric convection approach of 3-D reconstruction. In: Proc. 1st Symposium on Geometry Processing, pp. 218-229 (2003)

5. Chazal, F., Lieutier, A.: The $\lambda$-medial axis. In: Graphical Models (GMOD), vol. 67(4), pp. 304-331 (2005)

6. Chazal, F., Lieutier, A.: Weak feature size and persistent homology: computing homology of solids in $\mathbb{R}^{n}$ from noisy data samples. In: Proc. 21st ACM Sympos. Comput. Geom., pp. 255-262 (2005)

7. Chazal, F., Lieutier, A.: Topology guaranteeing manifold reconstruction using distance function to noisy data. In: Proc. 22st ACM Sympos. Comput. Geom., pp. 255-262 (2006)

8. Chazal, F., Soufflet, R.: Stability and finiteness properties of medial axis and skeleton. J. Dyn. Control Syst. 10(2), 149-170 (2004)

9. Cheeger, J.: Critical points of distance functions and applications to geometry. In: Geometric Topology: Recent Developments, Montecatini Terme, 1990. Springer Lecture Notes, vol. 1504, pp. 1-38 (1991)

10. Clarke, F.H.: Optimization and NonSmooth Analysis. Wiley, New York (1983)

11. Cohen-Steiner, D., Edelsbrunner, H., Harer, J.: Stability of persistence diagrams. In: Proc. 21st ACM Sympos. Comput. Geom., pp. 263-271 (2005)

12. Dey, T.K., Goswami, S.: Provable surface reconstruction from noisy samples. In: Proc. 20th ACM Sympos. Comput. Geom., pp. 330-339 (2004)

13. Dey, T.K., Wenger, R.: Reconstructing curves with sharp corners. In: Proc. 16th ACM Sympos. Comput. Geom., pp. 233-241 (2000)

14. Dey, T.K., Zhao, W.: Approximate medial axis as a Voronoi subcomplex. In: Proc. 7th ACM Sympos. Solid Modeling Applications, pp. 356-366 (2002)

15. Dey, T.K., Giesen, J., Ramos, E.A., Sadri, B.: Critical points of the distance to an epsilon-sampling of a surface and flow-complex-based surface reconstruction. In: Proc. 21st ACM Sympos. Comput. Geom., pp. 218-227 (2005)

16. Edelsbrunner, H.: Surface reconstruction by wrapping finite point sets in space. In: Aronov, B., Basu, S., Pach, J., Sharir, M. (eds.) Ricky Pollack and Eli Goodman Festschrift, pp. 379-404. Springer, New York (2002)

17. Edelsbrunner, H., Mücke, E.P.: Three-dimensional alpha shapes. ACM Trans. Graph. 13, 43-72 (1994)

18. Federer, H.: Curvature measures. Trans. Am. Math. Soc. 93, 418-419 (1959)

19. Foskey, M., Lin, M.C., Manocha, D.: Efficient computation of a simplified medial axis. In: ACM Symposium on Solid Modeling and Applications, pp. 96-107 (2003)

20. Giesen, J., John, M.: The flow complex: A data structure for geometric modeling. In: Proc. 14th Annual ACM-SIAM Symposium on Discrete Algorithms (SODA), pp. 285-294 (2003)

21. Grove, K.: Critical point theory for distance functions. In: Proc. of Symp. in Pure Mathematics, vol. 54, Part 3 (1993)

22. Lieutier, A.: Any open bounded subset of $\mathbb{R}^{n}$ has the same homotopy type as its medial axis. Comput.Aided Des. 36, 1029-1046 (2004). Elsevier

23. Mederos, B., Amenta, N., Velho, L., de Figueiredo, L.H.: Surface reconstruction from noisy point clouds. In: Proc. of Geometry Processing 2005 (Eurographics/ ACM SIGGRAPH), pp. 53-62

24. Niyogi, P., Smale, S., Weinberger, S.: Finding the homology of submanifolds with high confidence from random samples. Preprint available at http://www.tti-c.org/smale_papers.html

25. Robins, V., Meiss, J.D., Bradley, L.: Computing connectedness: Disconnectedness and discreteness. Physica D 139, 276-300 (2000)

26. Ruppert, J.: A Delaunay refinement algorithm for quality 2-dimensional mesh generation. J. Algorithms 18(3), 548-585 (1995)

27. Starck, J.L., Martínez, V.J., Donoho, D.L., Levi, O., Querre, P., Saar, E.: Analysis of the spatial distribution of galaxies by multiscale methods. arXiv:astro-ph/0406425 v1 (18 Jun. 04) 